GLASNIK MATEMATIČKI

Vol. 39(59)(2004), $199-205$

\title{
BOUNDS FOR THE SIZE OF SETS WITH THE PROPERTY $D(n)$
}

\author{
ANDREJ DUJELla \\ University of Zagreb, Croatia
}

\begin{abstract}
Let $n$ be a nonzero integer and $a_{1}<a_{2}<\cdots<a_{m}$ positive integers such that $a_{i} a_{j}+n$ is a perfect square for all $1 \leq i<j \leq m$. It is known that $m \leq 5$ for $n=1$. In this paper we prove that $m \leq 31$ for $|n| \leq 400$ and $m<15.476 \log |n|$ for $|n|>400$.
\end{abstract}

\section{INTRODUCTION}

Let $n$ be a nonzero integer. A set of $m$ positive integers $\left\{a_{1}, a_{2}, \ldots, a_{m}\right\}$ is called a $D(n)$-m-tuple (or a Diophantine $m$-tuple with the property $D(n)$ ) if $a_{i} a_{j}+n$ is a perfect square for all $1 \leq i<j \leq m$.

Diophantus himself found the $D(256)$-quadruple $\{1,33,68,105\}$, while the first $D(1)$-quadruple, the set $\{1,3,8,120\}$, was found by Fermat (see $[4,5])$. In 1969, Baker and Davenport [1] proved that this Fermat's set cannot be extended to a $D(1)$-quintuple, and in 1998, Dujella and Pethö [10] proved that even the Diophantine pair $\{1,3\}$ cannot be extended to a $D(1)$-quintuple. A famous conjecture is that there does not exist a $D(1)$-quintuple. We proved recently that there does not exist a $D(1)$-sextuple and that there are only finitely many, effectively computable, $D(1)$-quintuples (see [7, 9]).

The question is what can be said about the size of sets with the property $D(n)$ for $n \neq 1$. Let us mention that Gibbs [12] found several examples of Diophantine sextuples, e.g. $\{99,315,9920,32768,44460,19534284\}$ is a $D(2985984)$-sextuple.

Define

$$
M_{n}=\sup \{|S|: S \text { has the property } D(n)\} .
$$

2000 Mathematics Subject Classification. 11D45, 11D09, 11N36.

Key words and phrases. Diophantine $m$-tuples, property $D(n)$, large sieve. 
Considering congruences modulo 4 , it is easy to prove that $M_{n}=3$ if $n \equiv 2$ $(\bmod 4)($ see $[3,13,15])$. On the other hand, if $n \not \equiv 2(\bmod 4)$ and $n \notin$ $\{-4,-3,-1,3,5,8,12,20\}$, then $M_{n} \geq 4$ (see [6]).

In [8], we proved that $M_{n} \leq 32$ for $|n| \leq 400$ and

$$
M_{n}<267.81 \log |n|(\log \log |n|)^{2} \text { for }|n|>400 .
$$

The purpose of the present paper is to improve this bound for $M_{n}$, specially in the case $|n|>400$. We will remove the factor $(\log \log |n|)^{2}$, and also the constants will be considerably smaller.

The above mentioned bounds for $M_{n}$ were obtained in [8] by considering separately three types (large, small and very small) of elements in a $D(n)-m$ tuple. More precisely, let

$$
\begin{aligned}
& A_{n}=\sup \left\{\left|S \cap\left[|n|^{3},+\infty\right\rangle\right|: S \text { has the property } D(n)\right\}, \\
& B_{n}=\sup \left\{\left|S \cap\left\langle n^{2},|n|^{3}\right\rangle\right|: S \text { has the property } D(n)\right\}, \\
& C_{n}=\sup \left\{\left|S \cap\left[1, n^{2}\right]\right|: S \text { has the property } D(n)\right\} .
\end{aligned}
$$

In [8], it was proved that $A_{n} \leq 21$ and $B_{n}<0.65 \log |n|+2.24$ for all nonzero integers $n$, while $C_{n}<265.55 \log |n|(\log \log |n|)^{2}+9.01 \log \log |n|$ for $|n|>400$ and $C_{n} \leq 5$ for $|n| \leq 400$. The combination of these estimates gave the bound for $M_{n}$.

In the estimate for $A_{n}$, a theorem of Bennett [2] on simultaneous approximations of algebraic numbers was used in combination with a gap principle, while a variant of the gap principle gave the estimate for $B_{n}$. The bound for $C_{n}$ (number of "very small" elements) was obtained using the Gallagher's large sieve method [11] and an estimate for sums of characters.

In the present paper, we will significantly improve the bound for $C_{n}$ using a result of Vinogradov on double sums of Legendre's symbols. Let us mention that Vinogradov's result, in a slightly weaker form, was used recently, in similar context, by Gyarmati [14] and Sárközy \& Stewart [17]. We will prove the following estimates for $C_{n}$.

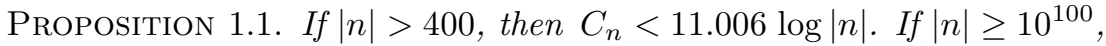
then $C_{n}<8.37 \log |n|$.

More detailed analysis of the gap principle used in [8] will lead us to the slightly improved bounds for $B_{n}$.

Proposition 1.2. For all nonzero integers $n$ it holds $B_{n}<0.6114 \log |n|+$ 2.158. If $|n|>400$, then $B_{n}<0.6071 \log |n|+2.152$.

By combining Propositions 1.1 and 1.2 with the above mentioned estimate for $A_{n}$, we obtain immediately the following estimates for $M_{n}$.

Theorem 1.3. If $|n| \leq 400$, then $M_{n} \leq 31$. If $|n|>400$, then $M_{n}<$ $15.476 \log |n|$. If $|n| \geq 10^{100}$, then $M_{n}<9.078 \log |n|$. 


\section{Three Lemmas}

Lemma 2.1 (Vinogradov). Let $p$ be an odd prime and $\operatorname{gcd}(n, p)=1$. If $A, B \subseteq\{0,1, \ldots, p-1\}$ and

$$
T=\sum_{x \in A} \sum_{y \in B}\left(\frac{x y+n}{p}\right)
$$

then $|T|<\sqrt{p|A| \cdot|B|}$.

Proof. See [18, Problem V.8.c)].

LEMMA 2.2 (Gallagher). If all but $g(p)$ residue classes $\bmod p$ are removed for each prime $p$ in a finite set $\mathcal{S}$, then the number of integers which remain in any interval of length $N$ is at most

$$
\left(\sum_{p \in \mathcal{S}} \log p-\log N\right) /\left(\sum_{p \in \mathcal{S}} \frac{\log p}{g(p)}-\log N\right)
$$

provided the denominator is positive.

Proof. See [11].

Lemma 2.3. If $\{a, b, c\}$ is a Diophantine triple with the property $D(n)$ and $a b+n=r^{2}, a c+n=s^{2}, b c+n=t^{2}$, then there exist integers $e, x, y, z$ such that

$$
a e+n^{2}=x^{2}, \quad b e+n^{2}=y^{2}, \quad c e+n^{2}=z^{2}
$$

and

$$
c=a+b+\frac{e}{n}+\frac{2}{n^{2}}(a b e+r x y) .
$$

Proof. See [8, Lemma 3].

\section{Proof of Proposition 1.1}

Let $N \geq n^{2}$ be a positive integer. Since $|n|>400$, we have $N>1.6 \cdot 10^{5}$. Let $D=\left\{a_{1}, a_{2}, \ldots, a_{m}\right\} \subseteq\{1,2, \ldots, N\}$ be a Diophantine $m$-tuple with the property $D(n)$. We would like to find an upper bound for $m$ in term of $N$. We will use the Gallagher's sieve (Lemma 2.2). Let

$$
\mathcal{S}=\{p: p \text { is prime, } \operatorname{gcd}(n, p)=1 \text { and } p \leq Q\},
$$

where $Q$ is sufficiently large. For a prime $p \in \mathcal{S}$, let $C$ denotes the set of integers $b$ such that $b \in\{0,1,2, \ldots, p-1\}$ and there is at least one $a \in D$ such that $b \equiv a(\bmod p)$. Then $\left(\frac{x y+n}{p}\right) \in\{0,1\}$ for all distinct $x, y \in C$. Here $(\dot{\bar{p}})$ denotes the Legendre symbol. If $0 \in C$, then $\left(\frac{n}{p}\right)=1$. For a given 
$x \in C \backslash\{0\}$, we have $\left(\frac{x y_{0}+n}{p}\right)=0$ for at most one $y_{0} \in C$. If $y \neq x, y_{0}$, then $\left(\frac{x y+n}{p}\right)=1$. Therefore,

$$
\begin{aligned}
T & =\sum_{x, y \in C}\left(\frac{x y+n}{p}\right)=\sum_{x \in C}\left(\sum_{y \in C}\left(\frac{x y+n}{p}\right)\right) \\
& \geq \sum_{x \in C}(|C|-3) \geq|C|(|C|-3) .
\end{aligned}
$$

On the other hand, Lemma 2.1 implies

$$
T<|C| \cdot \sqrt{p} .
$$

Thus, $|C|<\sqrt{p}+3$ and we may apply Lemma 2.2 with

$$
g(p)=\min \{\lfloor\sqrt{p}\rfloor+3, p\} .
$$

Let us denote the numerator and denominator from (2.1) by $E$ and $F$, respectively. By [16, Theorem 9], we have

$$
E=\sum_{p \in \mathcal{S}} \log p-\log N<\theta(Q)<1.01624 Q
$$

The function $f(x)=\frac{\log x}{\min \{\sqrt{x}+3, x\}}$ is strictly decreasing for $x>25$. Also, if $Q \geq 118$, then $f(p) \geq f(Q)$ for all $p \leq Q$.

For $p \in \mathcal{S}$ it holds $\operatorname{gcd}(n, p)=1$. This condition comes from the assumptions of Lemma 2.1. However, we will show later that $n$ can be divisible only by a small proportion of the primes $\leq Q$. Assume that $n$ is divisible by at most $5 \%$ of primes $\leq Q$. Then, for $Q \geq 118$, we have

$$
\begin{aligned}
F & \geq \sum_{p \in \mathcal{S}} f(p)-\log N \geq \frac{\log Q}{\sqrt{Q}+3} \cdot|\mathcal{S}|-\log N \\
& \geq \frac{\log Q}{\sqrt{Q}+3} \cdot \frac{19}{20} \pi(Q)-\log N>\frac{0.95 Q}{\sqrt{Q}+3}-\log N .
\end{aligned}
$$

Since $F$ has to be positive in the applications of Lemma 2.2, we will choose $Q$ of the form

$$
Q=c_{1} \cdot \log ^{2} N
$$

We have to check whether our assumption on the proportion of primes which divide $n$ is correct. Suppose that $n$ is divisible by at least $5 \%$ of the primes $\leq Q$. Then $|n| \geq p_{1} p_{2} \cdots p_{\lceil\pi(Q) / 20\rceil}$, where $p_{i}$ denotes the $i$-th prime. By $\left[16,3.5\right.$ and 3.12], we have $p_{\lceil\pi(Q) / 20\rceil}>R$, where

$$
R=\frac{1}{20} \frac{Q}{\log Q} \log \left(\frac{1}{20} \frac{Q}{\log Q}\right) .
$$


Assume that $c_{1} \geq 6$. Then $Q>860$ and $R>11.77$. From [16, 3.16], it follows that

$$
\log |n|>\sum_{p \leq R} \log p>R\left(1-\frac{1.136}{\log R}\right) .
$$

Furthermore, $\frac{1}{20} \frac{Q}{\log Q}>Q^{0.273}$ and $R>0.0136 Q$. Hence, (3.2) implies $\log R>7.793$ and therefore

$$
\log N \geq 2 \log |n|>0.01466 Q \geq 0.08796 \log ^{2} N,
$$

contradicting the assumption that $N>1.6 \cdot 10^{5}$.

Therefore, we have that $n$ is divisible by at most $5 \%$ of the primes $\leq Q$, and hence we have justified the estimate (3.1).

Under the assumption that $c_{1} \geq 6$, the inequality (3.1) implies

$$
F>0.861 \sqrt{Q}-\log N=\left(0.861 \sqrt{c_{1}}-1\right) \log N
$$

and

$$
\frac{E}{F}<\frac{1.017 c_{1}}{0.861 \sqrt{c_{1}}-1} \cdot \log N
$$

For $c_{1}=6$ we obtain

$$
\frac{E}{F}<5.503 \log N
$$

Assume now that $N \geq 10^{200}$ and $c_{1} \geq 4$. Then $Q>848303$ and we can prove in the same manner as above that $n$ is divisible by at most $1 \%$ of the primes $\leq Q$. This fact implies

$$
\frac{E}{F}<\frac{1.017 c_{1}}{0.986 \sqrt{c_{1}}-1} \cdot \log N
$$

For $c_{1}=4.11$ we obtain

$$
\frac{E}{F}<4.185 \log N
$$

Setting $N=n^{2}$ in (3.3) and (3.4), we obtain the statements of Proposition 1.1 .

\section{Proof of Proposition 1.2}

We may assume that $|n|>1$. Let $\{a, b, c, d\}$ be a $D(n)$-quadruple such that $n^{2}<a<b<c<d$. We apply Lemma 2.3 on the triple $\{b, c, d\}$. Since $b>n^{2}$ and $b e+n^{2} \geq 0$, we have that $e \geq 0$. If $e=0$, then $d=$ $b+c+2 \sqrt{b c+n}<2 c+2 \sqrt{c(c-1)+n}<4 c$, contradicting the fact that $d>4.89 c$ (see [8, Lemma 5]).

Hence $e \geq 1$ and

$$
d>b+c+\frac{2 b c}{n^{2}}+\frac{2 t \sqrt{b c}}{n^{2}} .
$$


Lemma 2.3 also implies

$$
c \geq a+b+2 r .
$$

From $r^{2} \geq a b-\sqrt[4]{a b}$ and $a b \geq 30$ it follows that $r>0.96 a$, and (4.2) implies $c>3.92 a$. Similarly, $b c \geq 42$ implies $t>0.969 \sqrt{b c}$ and, by (4.1), $d>b+c+3.938 \frac{b c}{n^{2}}>4.938 c+b$.

Assume now that $\left\{a_{1}, a_{2}, \ldots, a_{m}\right\}$ is a $D(n)$-m-tuple and $n^{2}<a_{1}<a_{2}<$ $\cdots<a_{m}<|n|^{3}$. We have

$$
a_{3}>3.92 a_{1}, \quad a_{i}>4.938 a_{i-1}+a_{i-2}, \quad \text { for } i=4,5, \ldots, m .
$$

Therefore, $a_{m}>\alpha_{m} a_{1}$, where the sequence $\left(\alpha_{k}\right)$ is defined by

$$
\alpha_{k}=4.938 \alpha_{k-1}+\alpha_{k-2}, \quad \alpha_{2}=1, \alpha_{3}=3.92 .
$$

Solving the recurrence (4.3), we obtain $\alpha_{k} \approx \beta \gamma^{k-3}$, with $\beta \approx 3.964355$, $\gamma \approx 5.132825$. More precisely,

$$
\left|\alpha_{k}-\beta \gamma^{k-3}\right|<\frac{1}{\beta \gamma^{k-3}} .
$$

From $|n|^{3}-1 \geq a_{m}>\alpha_{m} a_{1} \geq \alpha_{m}\left(n^{2}+1\right)$, it follows $\alpha_{m} \leq|n|-\frac{1}{|n|}$ and $\beta \gamma^{m-3}<|n|$. Hence,

$$
m<\frac{1}{\log \gamma} \log |n|+3-\frac{\log \beta}{\log \gamma} .
$$

For the above values of $\beta$ and $\gamma$ we obtain

$$
m<0.6114 \log |n|+2.158 .
$$

Assume now that $|n|>400$. Then $b c>a b>400^{4}$, which implies $c>$ $3.999999 a$ and $d>4.999999 c+b$. Therefore, in this case the relation (4.4) holds with $\beta \approx 4.042648, \gamma \approx 5.192581$, and we obtain

$$
m<0.6071 \log |n|+2.152 \text {. }
$$

REMARK 4.1. The constants in Theorem 1.3 can be improved, for large $|n|$, by using formula (2.26) from [16] in the estimate for the sum $\sum_{p \in \mathcal{S}} f(p)$. In that way, it can be proved that for every $\varepsilon>0, F>(2-\varepsilon) \sqrt{Q}-\log N$ holds for sufficiently large $Q$.

Also, in the proof of Proposition 1.2, for sufficiently large $|n|$ we have $c>$ $(4-\varepsilon) a$ and $d>(5-\varepsilon) c+b$, which leads to $B_{n}<\left(\frac{1}{\log \left(\frac{5+\sqrt{29}}{2}\right)}+\varepsilon\right) \log |n|$.

These results imply that for every $\varepsilon>0$ there exists $n(\varepsilon)$ such that for $|n|>n(\varepsilon)$ it holds

$$
M_{n}<\left(2+\frac{1}{\log \left(\frac{5+\sqrt{29}}{2}\right)}+\varepsilon\right) \log |n| .
$$




\title{
ACKNOWLEDGEMENTS.
}

The author is grateful to the referees for valuable comments, and in particular for pointing out a gap in the proof of Proposition 1.1 in the first version of the manuscript.

\section{REFERENCES}

[1] A. Baker and H. Davenport, The equations $3 x^{2}-2=y^{2}$ and $8 x^{2}-7=z^{2}$, Quart. J. Math. Oxford Ser. (2) 20 (1969), 129-137.

[2] M. A. Bennett, On the number of solutions of simultaneous Pell equations, J. Reine Angew. Math. 498 (1998), 173-199.

[3] E. Brown, Sets in which $x y+k$ is always a square, Math. Comp. 45 (1985), 613-620.

[4] L. E. Dickson, History of the Theory of Numbers, Vol. 2, Chelsea, New York, 1966, pp. 513-520.

[5] Diophantus of Alexandria, Arithmetics and the Book of Polygonal Numbers, (I. G. Bashmakova, Ed.), Nauka, Moscow, 1974 (in Russian), pp. 103-104, 232.

[6] A. Dujella, Generalization of a problem of Diophantus, Acta Arith. 65 (1993), 15-27.

[7] A. Dujella, An absolute bound for the size of Diophantine $m$-tuples, J. Number Theory 89 (2001), 126-150.

[8] A. Dujella, On the size of Diophantine m-tuples, Math. Proc. Cambridge Philos. Soc. 132 (2002), 23-33.

[9] A. Dujella, There are only finitely many Diophantine quintuples, J. Reine Angew. Math. 566 (2004), 183-214.

[10] A. Dujella and A. Pethő, A generalization of a theorem of Baker and Davenport, Quart. J. Math. Oxford Ser. (2) 49 (1998), 291-306.

[11] P. X. Gallagher, A larger sieve, Acta Arith. 18 (1971), 77-81.

[12] P. Gibbs, Some rational Diophantine sextuples, preprint, math.NT/9902081.

[13] H. Gupta and K. Singh, On k-triad sequences, Internat. J. Math. Math. Sci. 5 (1985), 799-804.

[14] K. Gyarmati, On a problem of Diophantus, Acta Arith. 97 (2001), 53-65.

[15] S. P. Mohanty and A. M. S. Ramasamy, On $P_{r, k}$ sequences, Fibonacci Quart. 23 (1985), 36-44.

[16] J. B. Rosser and L. Schoenfeld, Approximate formulas for some functions of prime numbers, Illinois J. Math. 6 (1962), 64-94.

[17] A. Sárközy and C. L. Stewart, On prime factors of integers of the form $a b+1$, Publ. Math. Debrecen 56 (2000), 559-573.

[18] I. M. Vinogradov, Elements of Number Theory, Nauka, Moscow, 1972 (in Russian).

\author{
A. Dujella \\ Department of Mathematics \\ University of Zagreb \\ Bijenička cesta 30, 10000 Zagreb \\ Croatia \\ E-mail: duje@math.hr \\ Received: 28.05.2003. \\ Revised: 22.09.2003.
}

